\title{
Effect of oxygen pressure on the structural and optical properties of $\mathrm{BaSnO}_{3}$ films prepared by pulsed laser deposition method.
}

JOHN, J., CHALANA, S.R., PRABHU, R. and PILLAI, V.P.M. 
Effect of Oxygen pressure on the structural and optical properties of $\mathrm{BaSnO}_{3}$ films prepared by pulsed laser deposition method

\author{
Jibi John' ${ }^{1}$, Chalana S. R ${ }^{1}$, Radhakrishna Prabhu² and V. P. Mahadevan Pillai ${ }^{1 *}$ \\ ${ }^{1}$ Department of Optoelectronics, University of Kerala, Kariavattom 695581,Thiruvanthapuram, Kerala, India. \\ ${ }^{2}$ School of Engineering, Robert Gordon University, Aberdeen, UK. \\ *E-mail:vpmpillai9@gmail.com.
}

\begin{abstract}
$\mathrm{BaSnO}_{3}$ thin films were prepared on quartz substrate by pulsed laser deposition technique under different background oxygen pressure. The effects of oxygen pressure on the structural, morphological and optical properties of $\mathrm{BaSnO}_{3}$ thin films were investigated using techniques such as X-ray diffraction (XRD), Scanning Electron Microscopic image analysis (SEM), energy-dispersive x-ray (EDX) spectroscopy, Ultraviolet-Visible (UV-Vis) spectroscopy and X-ray Photoelectron Spectroscopy (XPS). All the films were annealed at $700{ }^{\circ} \mathrm{C}$ for $1 \mathrm{hr}$ and the laser energy used is $60 \mathrm{~mJ}$. XRD analysis reveals that there is a systematic increase of intensity of $<110>$ with increase in oxygen pressure up to 0.02 mbar and thereafter shows reduction in intensity of the peak. A SEM and AFM image also reveals that the 0.02 mbar oxygen pressure has good morphology. XPS reveals that the presence of constituent elements in the prepared sample. The prepared sample has high transmittance in the visible region and infrared region.
\end{abstract}

\title{
Introduction
}

Perovskites with the general formula $\mathrm{MSnO}_{3}(\mathrm{M}=\mathrm{Ba}, \mathrm{Sr}$, $\mathrm{Ca}$ and $\mathrm{Pb})$ have found wide variety of applications [1]. Barium stannate $\left(\mathrm{BaSnO}_{3}\right)$ is a cubic perovskite oxide compound [2] that behaves as an n-type semiconductor [3] with a wide band gap of $3.4 \mathrm{eV}$ [3-6]. $\mathrm{BaSnO}_{3}$ has three-dimensional framework of corner-sharing $\mathrm{SnO}_{6}$ octahedra, having a linear Sn-O-Sn bonding angle $180^{\circ}$ with ideal cubic Pm3m space group [7]. By doping, with suitable dopants materials the properties of the $\mathrm{BaSnO}_{3}$ can be modified in a wide range due to its high substitutional flexibility [8,9]. $\mathrm{BaSnO}_{3}$ is used to prepare capacitors because of its characteristic dielectric properties [10]. It is also used as a sensor material for a wide variety of gases, such as $\mathrm{CO}, \mathrm{Cl}_{2}, \mathrm{NO}_{x}, \mathrm{O}_{2}, \mathrm{CO}_{2}, \mathrm{C}_{2} \mathrm{H}_{5} \mathrm{OH}, \mathrm{CH}_{3} \mathrm{SH}$ and sensing humidity and liquefied petroleum gas [11,12]. The preparation of $\mathrm{BaSnO}_{3}$ powder by conventional solidstate reaction through sintering $\mathrm{BaCO}_{3}$ and $\mathrm{SnO}_{2}$ at a high temperature ranging from $1,200{ }^{\circ} \mathrm{C}$ to $1,400{ }^{\circ} \mathrm{C}$ was reported by $\mathrm{S}$. Upadhyay 
et.al. [13,14]. Hydrothermal synthesis route for the preparation of $\mathrm{BaSnO}_{3}$ is reported by Kutty and Vivekanadan [15]. Mildly lanthanum doped $\mathrm{BaSnO}_{3}$ exhibits very high electrical mobility of $320 \mathrm{~cm}^{2}(\mathrm{Vs})^{-1}$ at room temperature and superior thermal stability at high temperatures [16]. Lampe et al [11] reported the NO and CO sensing behaviour of $\mathrm{BaSnO}_{3}$ thin films at the temperature range of $450-550{ }^{\circ} \mathrm{C}$, and of $600-700{ }^{\circ} \mathrm{C}$ respectively [17]. The preparation of lanthanum doped $\mathrm{BaSnO}_{3}$ with substrate temperature $850{ }^{\circ} \mathrm{C}$ and oxygen partial pressure $1 * 10^{-3}$ Torr using pulsed laser deposition is reported by Sallis et al. [18]. A carrier concentration of $2 * 10^{21} \mathrm{~cm}^{-3}$ and mobility $0.69 \mathrm{~cm}^{2} / \mathrm{Vs}$ was reported in lanthanum-doped barium stannate films [19]. Although there are different techniques reported for thin film preparation the pulsed laser deposition (PLD) method is simple and unique technique which can be used to prepare good quality films from a large amount of materials and compounds [20]. In this method one can vary substrate temperature, background pressure, substrate to target distance, laser fluence, etc.. PLD is one of the best methods for preparing the films which maintains the composition close to that of target [20]. There are a large amount of studies in bulk and doped $\mathrm{BaSnO}_{3}$ powder, but studies in thin films of $\mathrm{BaSnO}_{3}$ are very rarely reported. Thin films of iron and lanthanum doped barium stannate is reported by Jayaraj et al and its magnetic properties are studied [21,22]. The electrical properties of $\mathrm{BaSnO}_{3}$ doped lanthanum thin films by radio frequency magnetron sputtered method are reported by Lou et al [23]. This paper reports $\mathrm{BaSnO}_{3}$ thin films prepared by pulsed laser deposition technique and a study on the effect of background oxygen pressure on its structural, morphological and its optical properties.

\section{Experiment}

$\mathrm{BaSnO}_{3}$ powder was prepared by the conventional high temperature solid state method [13,14]. High quality (purity99.99\%, Sigma) $\mathrm{BaCO}_{3}$ and $\mathrm{SnO}_{2}$ powders are mixed in stoichiometric ratio and the mixture was ground well in an agate mortar for 6 hours using acetone as the mixing agent. The grinded powder taken in a platinum crucible was heated in a microprocessor programmable furnace for $6 \mathrm{~h}$ at a temperature of $1250^{\circ} \mathrm{C}$. During heating the rate of rise of temperature was kept at $5^{\circ} \mathrm{C} / \mathrm{min}$. After heating, the powder was cooled to room temperature naturally. The phase purity of the $\mathrm{BaSnO}_{3}$ powder thus prepared was analyzed by the $\mathrm{X}$ ray diffraction pattern and micro-Raman spectrum. The $\mathrm{BaSnO}_{3}$ powder thus prepared was well-ground in an agate mortar and poly vinyl alcohol was added to the mixture as the binding agent. Pellets of $3 \mathrm{~mm}$ diameter and $1 \mathrm{~mm}$ thickness were prepared from the $\mathrm{BaSnO}_{3}$ powder in a hydraulic press by applying pressure of 8 ton and the pressed pellet was sintered at temperature of $1000^{\circ} \mathrm{C}$ for $6 \mathrm{~h}$ in a programmable furnace. The well-sintered pellet thus prepared is used as the target for film preparation. The 
deposition of $\mathrm{BaSnO}_{3}$ films was done in a multiport chamber which was pre-evacuated to a base pressure of $10^{-6} \mathrm{mbar}$ using a turbo molecular pump and a rotary pump. The ablation of target was carried out using alaser radiation at 355 nm wavelength (pulse width $7 \mathrm{~ns}$ and repetition rate 10Hz) from a Q switched (frequency tripled) Nd:YAG laser (Quanta Ray INDI Series-Spectra Physics). Cleaned quartz substrate kept at distance of $4.5 \mathrm{~cm}$ from the target was heated at a temperature of $600^{\circ} \mathrm{C}$. The films are fabricated on heated substrate for a deposition time of 45 min using a laser fluence of $60 \mathrm{~mJ}$ at different back ground oxygen pressures viz 0, 0.01, 0.015, 0.02, 0.025 and 0.03 mbar. To maintain uniform ablation during film preparation the target was rotated (speed $33 \mathrm{rpm}$ ). All the films are annealed at temperature of $700^{\circ} \mathrm{C}$ for $1 \mathrm{~h}$. The film prepared without oxygen ambience is designated as BS0 and the films prepared with background oxygen pressures $0.01,0.015,0.02,0.025$ and 0.03 mbar are designated as BS 0.01, BS0.015, BS0.02, BS0.025 and BS 0.03 respectively.

Crystalline structure of the $\mathrm{BaSnO}_{3}$ films was characterized by XPERT PRO diffractometer in the $2 \theta$ range $20-80^{\circ}$ using $\mathrm{Cu}-\mathrm{K} \alpha_{\mathrm{L}}$ radiation of wavelength $1.5406^{\circ} \mathrm{A}$ in Bragg Brentano geometry. The measurements were carried out with a step size of 0.04000 and a scan speed of $2^{\circ} \mathrm{min}^{-1}$ was maintained. Surface morphology of the films was analyzed using Atomic Force Microscopy (AFM) and Scanning Electron Microscopy (SEM) at nanometric scale using by Bruker, Dimension Edge with ScanAssist in contact mode and Nova Nano SEM-450 Field Emission Scanning Electron Microscope (FEI- USA) respectively. The thickness of the films was measured using lateral SEM analysis. Photoluminescence spectra of the films were recorded using Horiba Jobin Yvon Flourolog III modular spectroflourometer equipped with Xe-flash lamp using an excitation wavelength of $350 \mathrm{~nm}$. The optical absorption, transmission spectroscopic measurements of all the films were performed in the wavelength range of 2300-250 nm using Perkin Elmier Lambda 950 spectrophotometer. The surface composition and elemental analysis were done using X-ray Photoelectron Spectrometer (XPS) ESCALABX1+A1528 using avantage software.

\section{Results and discussion}

\section{XRD analysis}

XRD patterns of the laser ablated $\mathrm{BaSnO}_{3}$ films are shown in Fig.1 which indicate the polycrystalline nature of the films and all the XRD patterns present peaks corresponding to cubic $\mathrm{BaSnO}_{3}$ crystalline phase [JCPDS Ref. Code 15 -0780]. The XRD pattern of BS 0 film presents two high intense peaks at $2 \theta$ values $30.76^{\circ}$ and $44.04^{\circ}$, a medium 
intense peak at $54.67^{\circ}$ and weak peak at $2 \theta$ value $72.72^{\circ}$. These peaks can be indexed to lattice reflection planes (110), (200), (211) and (300) of cubic $\mathrm{BaSnO}_{3}$ crystalline phase respectively. In addition to these four peaks, a medium intense peak at $2 \theta$ value $64.05^{\circ}$ corresponding to (220) lattice reflection plane of cubic phase can be seen in the XRD patterns of the films prepared under background oxygen. In the XRD patterns of all the films, (110) plane possesses the highest intensity indicating $<110>$ as the preferred direction of crystalline growth in films. In films prepared under background oxygen pressure $\left(\rho \mathrm{O}_{2}\right)$, the intensity of (110) peak shows a systematic increase with increase in $\rho \mathrm{O}_{2}$ up to 0.02 mbar and thereafter the intensity of the peak decreases with increase in $\rho \mathrm{O}_{2}$ (Fig. 2a). The $\beta$ (hkl), Full Width at Half Maximum (FWHM), of the (110) peak is estimated from the XRD data and are given in Table 1 and for the BSO film its value is $0.262^{\circ}$. The value of $\beta(\mathrm{hkl})$ of (110) peak shows a systematic decrease with increase in background oxygen pressure up to $\rho \mathrm{O}_{2}=0.02$ mbar and thereafter it increases with increase in back ground oxygen pressure. From this it can be inferred that moderate oxygen ambience will be helpful in getting good crystalline $\mathrm{BaSnO}_{3}$ film and the film prepared at $\rho \mathrm{O}_{2}=0.02$ mbar possesses the highest crystalline quality.

From the XRD patterns of the films, it can be seen that as $\rho_{2}$ increases, both (110) and (200) peaks show large intensity enhancement up to $\rho \mathrm{O}_{2}=0.02 \mathrm{mbar}$. The relative intensities $\rho(110)$ and $\rho(200)$ of the peaks (110) and (200) for the films prepared under background oxygen pressure with respect to those values in BS0 films are estimated and are plotted in the Fig. 2b. This indicates that at low or moderate oxygen pressure there exists a competition for growing the crystallites along $<110>$ and $<200>$ directions. At higher oxygen pressures these competing tendency of crystal growth disappears and the crystallite prefer to grow along $<110>$ direction. The observed deterioration in the crystalline of the films at higher background oxygen pressures (>0.02mbar) may be due to the decrease in the deposition rate with increase in the background oxygen ambience [24]. Beyond 0.02mbar oxygen pressure, increase in the background oxygen pressure makes the mean free path shorter and it results in the enhanced scattering of the ejected particles in the laser produced plasma by the oxygen gas molecules. It may cause reduction in the rate of film formation and the deterioration of the intensity may be due to the prevention of deposition of the atoms from reaching the right position in the crystal lattice from the flux at higher oxygen pressure [25,26]. 

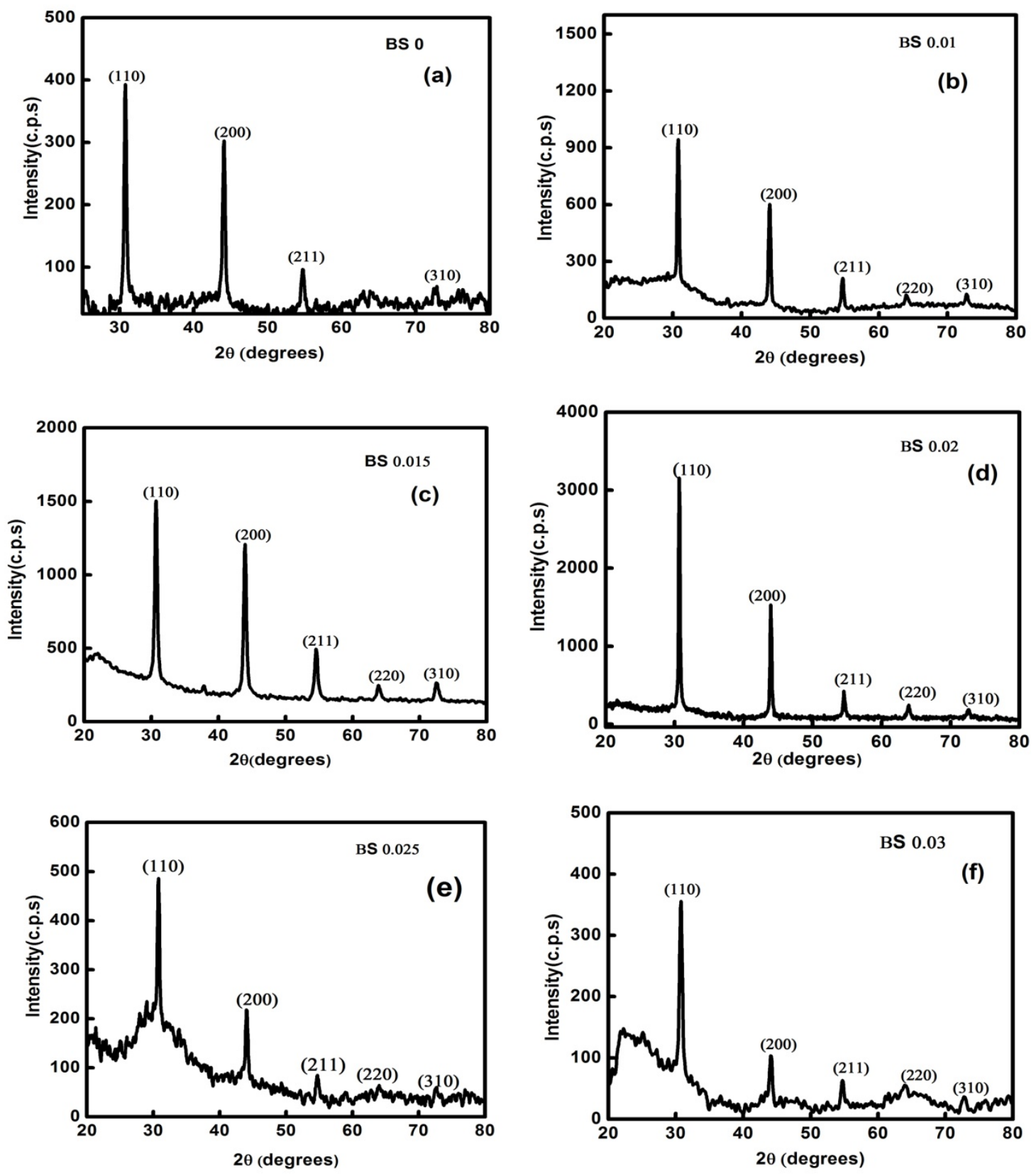

Fig.1. XRD patterns of laser ablated $\mathrm{BaSnO}_{3}$ thin films deposited on quartz substrate for different oxygen pressures

The average size of the crystallites ( $D_{h k l}$ ) of the films can be calculated using the Debye Scherer's formula [27], 


$$
D_{h k l}=\frac{k \lambda}{\beta_{h k l} \cos \theta_{h k l}}
$$

Table 1.Structural parameters of $\mathrm{BaSnO}_{3}$ thin films prepared under different oxygen pressures

\begin{tabular}{|c|c|c|c|c|c|c|c|}
\hline \multirow[t]{2}{*}{ sample } & \multirow[t]{2}{*}{$\rho \mathrm{I}_{(110)}$} & \multirow[t]{2}{*}{$\rho \mathrm{I}_{(200)}$} & \multirow{2}{*}{$\begin{array}{l}\beta(110) \\
\text { (degrees) }\end{array}$} & \multicolumn{2}{|c|}{ Crystalline size (nm) } & \multirow{2}{*}{$\begin{array}{l}\text { Lattice } \\
\text { constant } \\
(\mathrm{nm})\end{array}$} & \multirow[t]{2}{*}{ Lattice strain } \\
\hline & & & & $\begin{array}{l}\text { From } \\
\text { Scherer } \\
\text { Formula(D) }\end{array}$ & $\begin{array}{l}\text { From } \\
\text { WH } \\
\text { plot(D') }\end{array}$ & & \\
\hline BS 0 & 1 & 1 & 0.262 & 30 & $47 \pm 5$ & 0.4103 & $\begin{array}{l}0.00619 \pm 0.0 \\
0134\end{array}$ \\
\hline BS 0.01 & 2.56 & 2.17 & 0.256 & 32 & $63 \pm 18$ & 0.4104 & $\begin{array}{l}0.00286 \pm 0.0 \\
0126\end{array}$ \\
\hline BS 0.015 & 3.33 & 3.71 & 0.251 & 33 & $66 \pm 33$ & 0.4105 & $\begin{array}{l}0.00373 \pm 0.0 \\
0131\end{array}$ \\
\hline BS 0.02 & 6.8 & 4.62 & 0.221 & 34 & $69 \pm 23$ & 0.4105 & $\begin{array}{l}0.00282 \pm 0.0 \\
0203\end{array}$ \\
\hline BS 0.025 & 0.83 & 0.52 & 0.277 & 31 & $53 \pm 22$ & 0.4106 & $\begin{array}{l}0.00538 \pm 0.0 \\
022\end{array}$ \\
\hline BS 0.03 & 0.82 & 0.298 & 0.301 & 27 & $40 \pm 5$ & 0.4104 & $\begin{array}{l}0.00854 \pm 0.0 \\
0505\end{array}$ \\
\hline
\end{tabular}


Where $\lambda$ is the wavelength of the $\mathrm{X}$-ray radiation, $\mathrm{k}$ is a constant which can be taken as 0.9 for spherical crystallites and $\theta_{h k l}$ is the diffraction angle. The average size of the crystallites in the prepared films varies from 27 to $34 \mathrm{~nm}$ and is shown in Table1. The lattice constants for the films were calculated and found to be in agreement with the

reported value $4.101 \mathrm{~A}^{\circ}[28-30]$. 

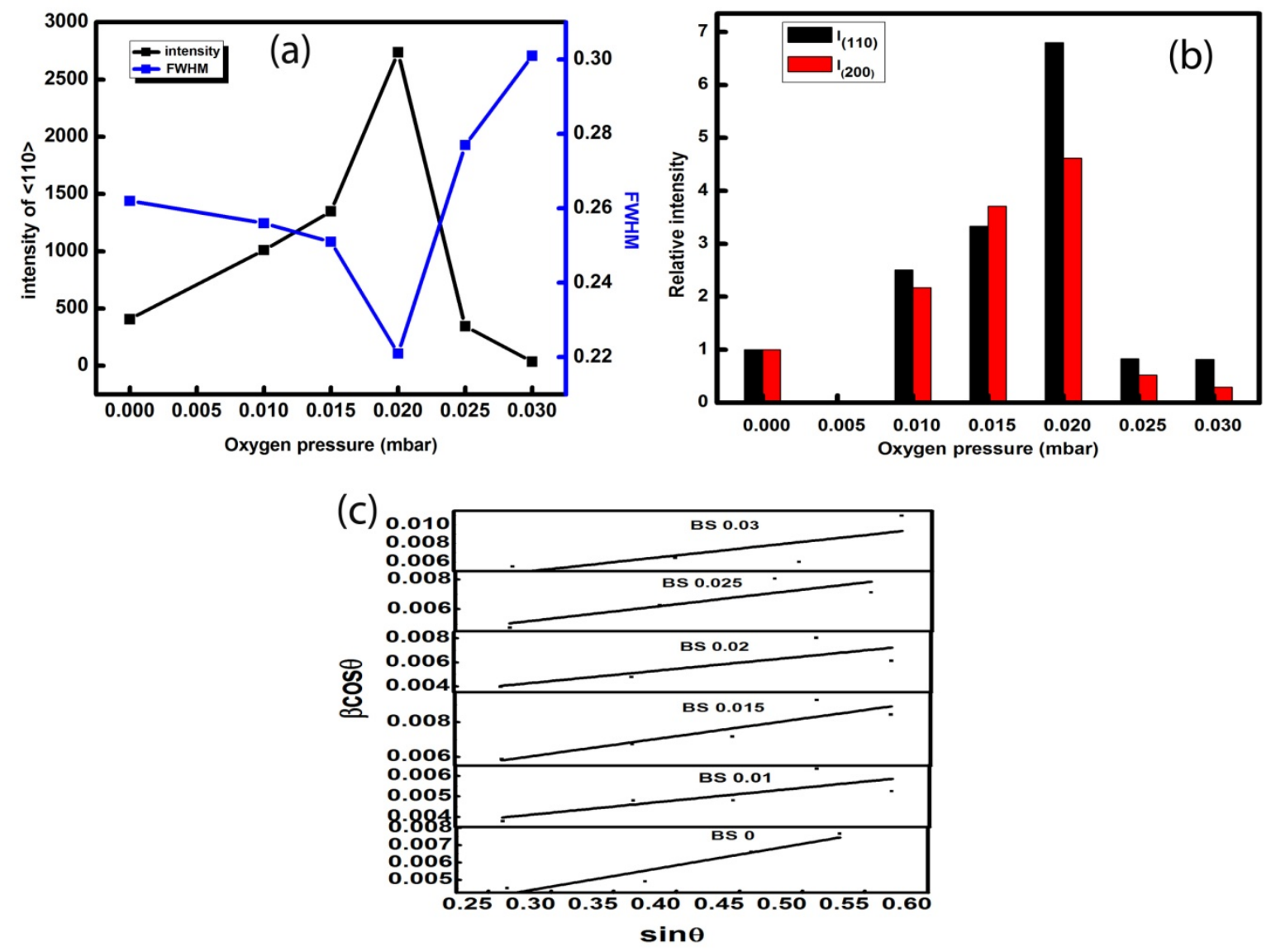

Fig. 2a.Variation of intensity and FWHM of 110 peak as a function of background oxygen pressures, 2b. Variation of relative intensity $\rho(110)$ ) and $\rho(200)$ ) as a function of background oxygen pressures and 2c. Williamson-Hall plots for $\mathrm{BaSnO}_{3}$ films deposited by pulsed laser deposition method on quartz substrate.

The factors such as the internal stress, thermal stress, strain and lattice distortion in the films can broaden the XRD peaks and hence the crystallite size estimated using the Scherer formula can be smaller than the actual value of the crystalline size $[31,32]$. The Williamson-Hall relation can be used to study the effect of strain induced broadening of crystallite size in the full width at half maximum (FWHM) of XRD peaks [33] and it is shown in Fig.2c.

$\beta_{h k l} \cos \theta_{h k l}=\frac{k \lambda}{\mathrm{D}^{\prime}}+2 \eta \sin \theta_{\mathrm{hkl}^{-}}$

$\mathrm{D}^{\prime}$ is the size of the strain free crystallite, and $\eta$ is the lattice strain. Plots of $\sin \theta$ vs. $\beta \cos \theta$ are drawn for all the films and straight line plots are obtained. The y-intercept of the plot is a measure of $k \lambda / D^{\prime}$ from which the crystallite size 
can be calculated and the strain can be estimated from the slope of the plot (Table 1). From the data shown in Table 1 it can be found that all the films prepared have considerable strain in them and there is strain induced broadening for the XRD peaks.

\section{XPS Analysis}

X-ray photoelectron spectroscopy (XPS) was used for the analysis of the chemical states and the composition of the elements present in the laser ablated $\mathrm{BaSnO}_{3}$ films prepared under oxygen ambience. The presence of the peaks corresponding to $\mathrm{Ba}, \mathrm{Sn}$ and $\mathrm{O}$ in the XPS spectra of the films suggest the formation of $\mathrm{BaSnO}_{3}$ phase in the films. The XPS survey spectrum of a typical $\mathrm{BaSnO}_{3}$ film deposited under oxygen ambience at $\rho_{2}=0.02 \mathrm{mbar}$ is shown in Fig.3a. The C1s peak at $284.4 \mathrm{eV}$ observed in the XPS spectrum of the film can be due to the carbon contamination of film surface in the presence of air. Fig.3b shows the core level spectra of Ba3d peaks of Ba. Manju et al reported Ba3d $_{3 / 2}$ and $\mathrm{Ba}_{5} \mathrm{~d}_{5 / 2}$ peaks at binding energy values 795.2 and $777.9 \mathrm{eV}$ with a peak separation of $15.3 \mathrm{eV}$ corresponding to $\mathrm{Ba}^{2+}$ state [34]. Singh et al reported the Ba3d spin orbit doublet peaks of $\mathrm{Ba}_{3 / 2}$ and $\mathrm{Ba}_{3 / 2}$ at the binding energies 794.8 and $779.4 \mathrm{eV}$ respectively with a peak separation of $15.4 \mathrm{eV}$ [35]. In the present case, for the BSO film, the core level spectrum of Ba (Fig. 3b) yields two peaks at binding energy values 795.06 and $779.78 \mathrm{eV}$ respectively with a peak difference of $15.28 \mathrm{eV}$. These peaks can be unambiougesly assigned to spin orbit doublet peaks of $\mathrm{Ba}_{3 / 2}$ and $\mathrm{Ba} 3 \mathrm{~d}_{5 / 2}$ respectively. In all the films prepared at different oxygen pressure namely $\rho \mathrm{O}_{2}=0.01$, 0.02 and 0.03 mbar, the $\mathrm{Ba}_{3 / 2}$ and $\mathrm{Ba}_{3 / 2}$ peak sare observed at binding energy values of 795.32 and $780.04 \mathrm{eV}$ respectively (with the peak difference of $15.28 \mathrm{eV}$ ). The observed peak positions suggest that $\mathrm{Ba}$ atom in the compound is in the $\mathrm{Ba}^{2+}$ state.

According to Kwoka et al., Sn can exist in three oxidization states $\mathrm{Sn}^{0}, \mathrm{Sn}^{2+}$ and $\mathrm{Sn}^{4+}$ corresponding to binding energies 485.0, 485.9 and $486.6 \mathrm{eV}$ respectively [36]. The Fig.3c shows the core level spectra of sn3ds tate of the different films. For the BS 0 film for the spin orbit doublets $S n 3 d_{5 / 2}$ and $S n 3 d_{3 / 2}$ peaks are observed at binding energy values of 486.04 and $494.49 \mathrm{eV}$ with peak difference of $8.45 \mathrm{eV}$. For all the films prepared under different oxygen pressures namely $\rho \mathrm{O}_{2}=0.01,0.02$ and 0.03 mbar (BS 0.01, BS 0.02 and BS 0.03 ), the Sn3d peaks are observed at binding energy values of $486.67 \mathrm{eV}$ and $495.12 \mathrm{eV}$ with the peak difference of $8.45 \mathrm{eV}$. The observed peak positions suggest that tin is in the $\mathrm{Sn}^{4+}$ state. The $\mathrm{O}$ 1s peak (Fig. 3d) is observed at $531.33 \mathrm{eV}$ for the $\mathrm{BS} 0$ film and $531.17 \mathrm{eV}$ for the films prepared under different oxygen pressures $0.01,0.02$ and 0.03 mbar. It is reported that the peak in the 
range 530.1 to $532.0 \mathrm{eV}$ corresponds to the oxygen vacancy $[37,38,39]$ This confirms the presence of oxygen vacancy or defects in the films.
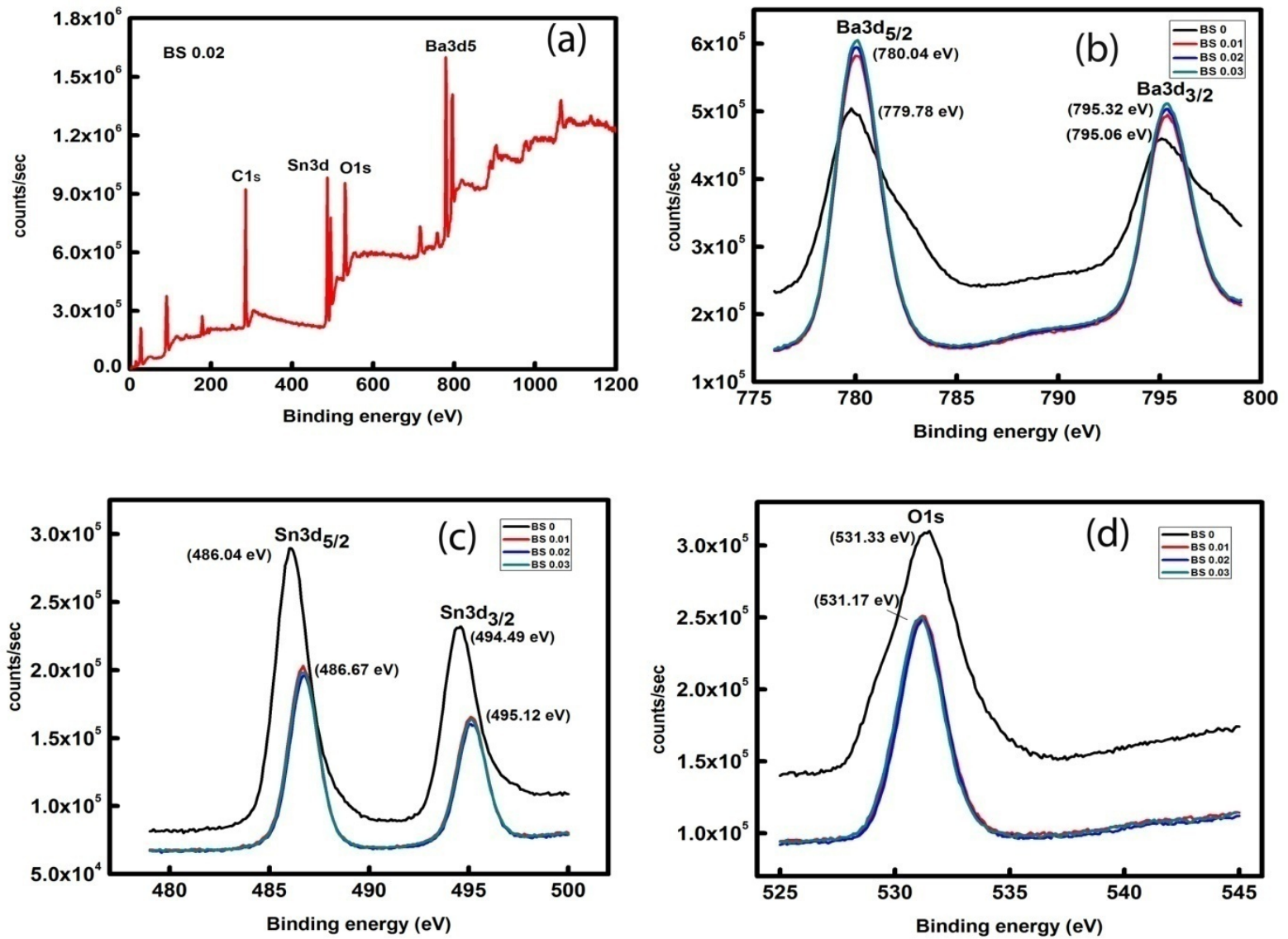

Fig.3 XPS spectra of laser ablated $\mathrm{BaSnO}_{3}$ film (a). XPS survey spectrum of BS 0.02 film, (b). the core level splitting of peaks of Ba, (c). the core level splitting of peaks of Sn and (d). the core level splitting of peaks of O.

\section{Morphological analysis}

The FESEM images of the films deposited without background oxygen ambience and with oxygen ambience are shown in Fig. 4. The effect of oxygen ambience on the surface morphology of the $\mathrm{BaSnO}_{3}$ films can be clearly seen in the FESEM images. The surface morphology of the BS0 film (Fig. 4a) shows the random distribution of isolated clusters of different sizes which are formed due to the agglomeration of smaller grains. The FESEM images of films 
prepared under the oxygen ambience (Figs. 4(b)-4(f)) show the uniform dense distribution of grains with good connectivity. This indicates that the films with better quality are formed in the presence of oxygen ambience.
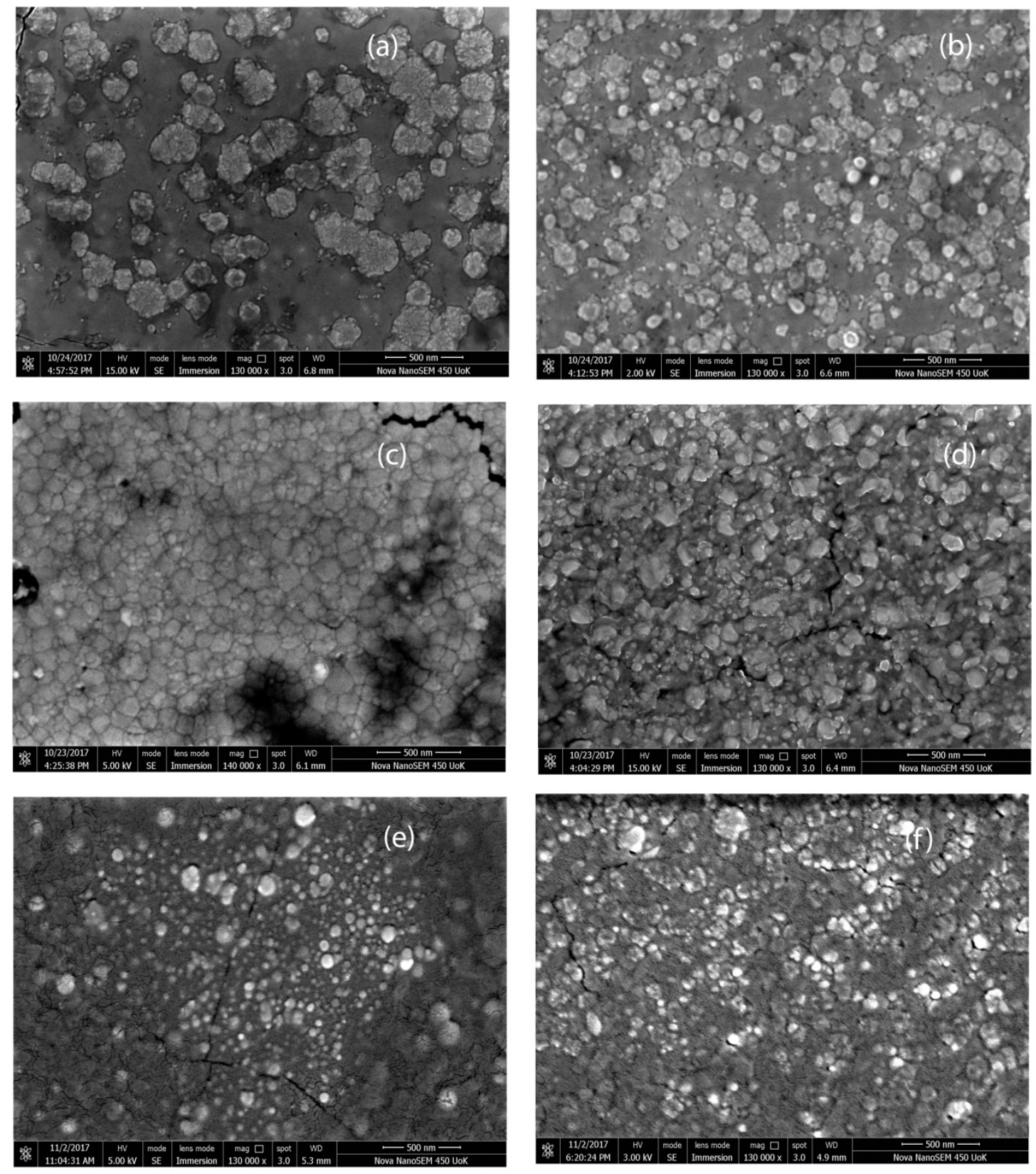

Fig.4. FESEM images of laser ablated $\mathrm{BaSnO}_{3}$ films deposited at different oxygen pressures, (a). film in oxygen free ambience, (b) film at $\rho \mathrm{O}_{2}=0.01 \mathrm{mbar}$, (c) film at $\rho \mathrm{O}_{2}=0.015 \mathrm{mbar}$, (d) film at $\rho \mathrm{O}_{2}=0.02$ mbar, (e) film at $\rho \mathrm{O}_{2}=$ 0.025 mbar and (f) film at $\rho \mathrm{O}_{2}=0.03$ mbar 

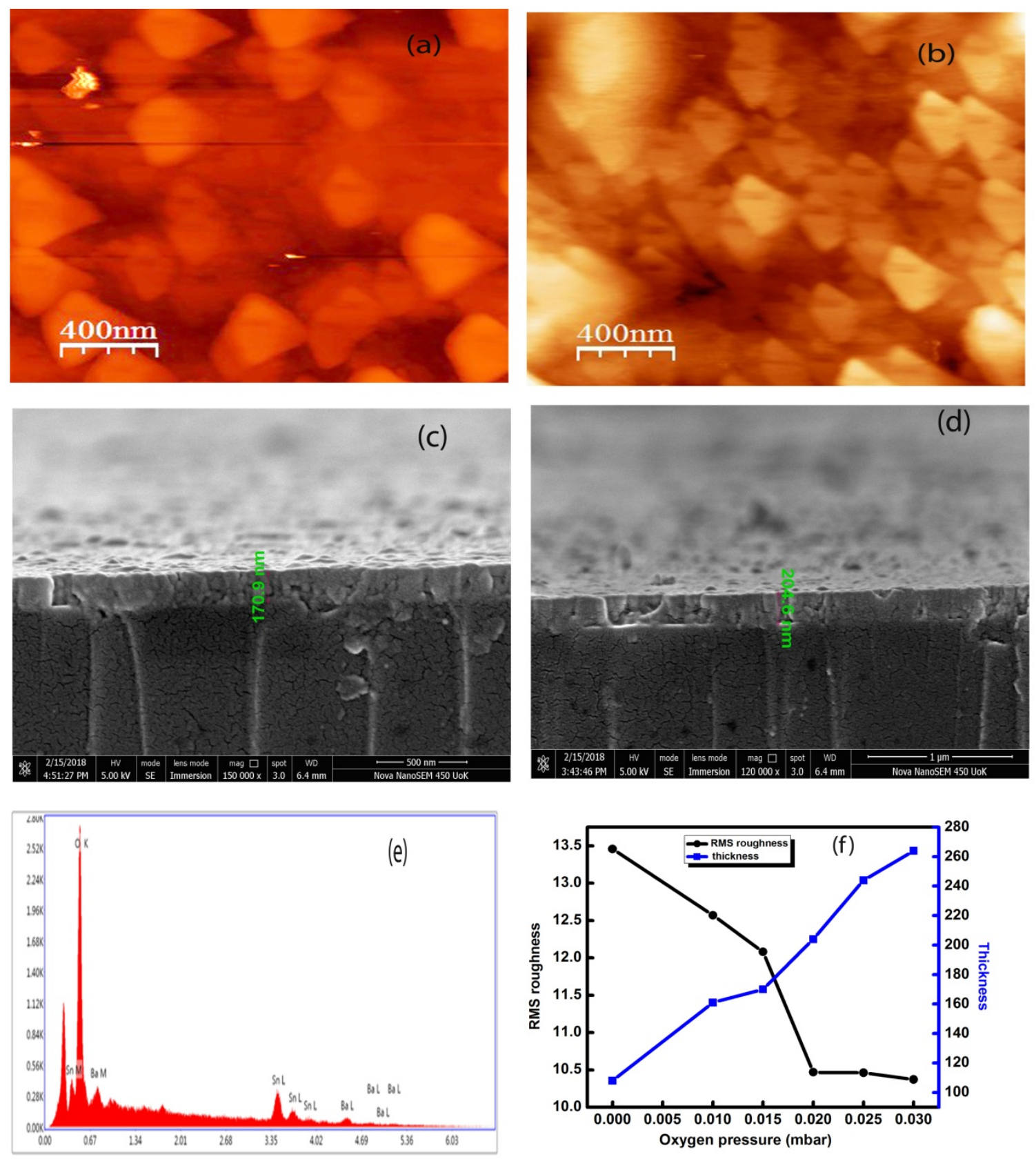

Fig.5(a). AFM image of BS 0.015 film, 5(b). AFM image of BS 0.02 film, 5(c). Cross sectional FESEM image of BS 0film, 5(d). Cross sectional FESEM image of BS 0.015 film 5(e) Cross sectional FESEM image of BS 0.02 film, and 5(f). Variations of RMS surface roughness and film thickness as a function of background oxygen pressure.

AFM analysis is useful for the study of the surface morphology, surface homogeneity and nanostructure of deposited films. The AFM images of two typical films namely BS 0.015 and BS 0.02 are shown in Figs. 5(a) and 5(b) 
respectively. The root mean square(RMS) surface roughness of the $\mathrm{BaSnO}_{3}$ films is obtained using WSXM 5.0 Develop 6.4 software and the values are shown in Table 2. It can be seen that RMS surface roughness decreases with increase in the value of background oxygen pressure. The bombarding species on the substrate and the film surface may vary with different oxygen pressures and hence the surface roughness can change with oxygen pressure [40]. This indicates the formation of the films with more uniform surface morphology, at higher oxygen pressures. The thickness of films is measured using the cross-sectional FESEM images. The cross sectional FESEM images of BS 0.015 and BS 0.02 are shown in Fig.5c and 5d. The non-uniform formation of film with less thickness can be seen in the FESEM image of BS0 film. But, the formation of films with uniform thickness can be seen in the cross sectional FESEM images of films deposited at background oxygen pressure 0.01 mbar and 0.02 mbar. Thus optimum value of background oxygen pressure is helpful in getting high quality uniform films. It is found that the thickness of the film increases systematically with increase in background oxygen pressures (Fig.5f). In the presence of background oxygen more number of scattering centers are available and they scatter the incoming flux so that The mobility and the diffusion of surface atoms will increase with increase in oxygen pressure. This may be the reason for the increase of film thickness with increase in oxygen pressure [41].

The elemental analyses are done using energy dispersive X-ray (EDX) spectra (Figures not shown). The presence of peaks corresponding to $\mathrm{Ba}, \mathrm{Sn}$ and $\mathrm{O}$ indicate the formation of $\mathrm{BaSnO}_{3}$ films.

Table 2.Optical and Morphological parameters of $\mathrm{BaSnO}_{3}$ thin films for different oxygen pressures

\begin{tabular}{|l|l|l|l|l|}
\hline Sample code & $\begin{array}{l}\text { Average } \\
\text { transmittance } \\
\mathbf{( \% )}\end{array}$ & $\begin{array}{l}\text { Band } \\
\mathbf{( e V )}\end{array}$ & $\begin{array}{l}\text { RMS roughness } \\
\mathbf{( n m )}\end{array}$ & $\begin{array}{l}\text { Thickness } \\
\mathbf{( n m})\end{array}$ \\
\hline BS 0 & 65 & 2.91 & 13.46 & 108 \\
\hline BS 0.01 & 81 & 3.04 & 12.57 & 161 \\
\hline BS 0.015 & 77 & 3.05 & 12.08 & 170 \\
\hline BS 0.02 & 85 & 3.09 & 10.47 & 204 \\
\hline BS 0.025 & 82 & 3.09 & 10.46 & 244 \\
\hline BS 0.03 & 84 & 3.06 & 10.37 & 264 \\
\hline
\end{tabular}




\section{Optical analysis}

The optical absorbance and transmittance spectra of the films deposited at different oxygen pressures in the wavelength range 190-2300 $\mathrm{nm}$ are shown in Figs. 6(a)-6(b) respectively. All the films have good transparency in the visible and near infrared range. The sharp fall of transmittance near the absorption edge observed for all the films indicate their good crystalline nature. The average transmittance of the films in the wavelength region 250-2300 nm are measured and the values are shown in Table 2. The BS 0 film present an average transmittance value of $65 \%$ whereas the films prepared under oxygen ambience possess higher values of average transmittance. This indicates the films prepared under oxygen ambience are of superior quality compared to that prepared in oxygen free ambience. The absorption coefficient $\alpha$, can be calculated from the transmittance spectra using the following relation $\alpha=\frac{1}{d} \ln \frac{1}{T} \quad-(3)$

where $d$ is the thickness of the film and $T$ is the transmittance of the film. The band gap energy $E_{g}$ of the can be estimated from the following relation [32]

$$
\alpha(h v)=A\left(h v-E_{g}\right)^{n} \quad-(3)
$$

$h$ is the Planck's constant, constant $A$ is the band edge sharpness which is related with the order in crystalline structure of deposited films, $v$ is the frequency of radiation, and the exponent $n$ is the exponent depending on the nature of band transition. The values of $n$ for direct allowed and direct forbidden transitions will be $1 / 2$ and $3 / 2$ respectively. For the indirect allowed and indirect forbidden transitions $n$ can take values 2 and 3 respectively $[32,42,43]$. The band gap is determined for all the films from the plot of $(\alpha)^{1 / n}$ versus photon energy $h v$ on the $x$ axis by extrapolating the linear portion of the graph to the $h v=0$. It is observed that for all the films, the best straight line is obtained for $n=2$, which corresponds to indirect allowed transition. The estimated values of the optical energy gap for the films varies from 2.91 to $3.09 \mathrm{eV}$ (Table 2).The optical energy gap for the $\mathrm{BS} 0$ film is $2.91 \mathrm{eV}$ and its value is higher in films prepared under oxygen ambience. The optical energy gap increases systematically with increase in $\rho \mathrm{O}_{2}$ up to 0.025 mbar oxygen pressure thereafter decreases slightly with increase in $\rho \mathrm{O}_{2}$. 

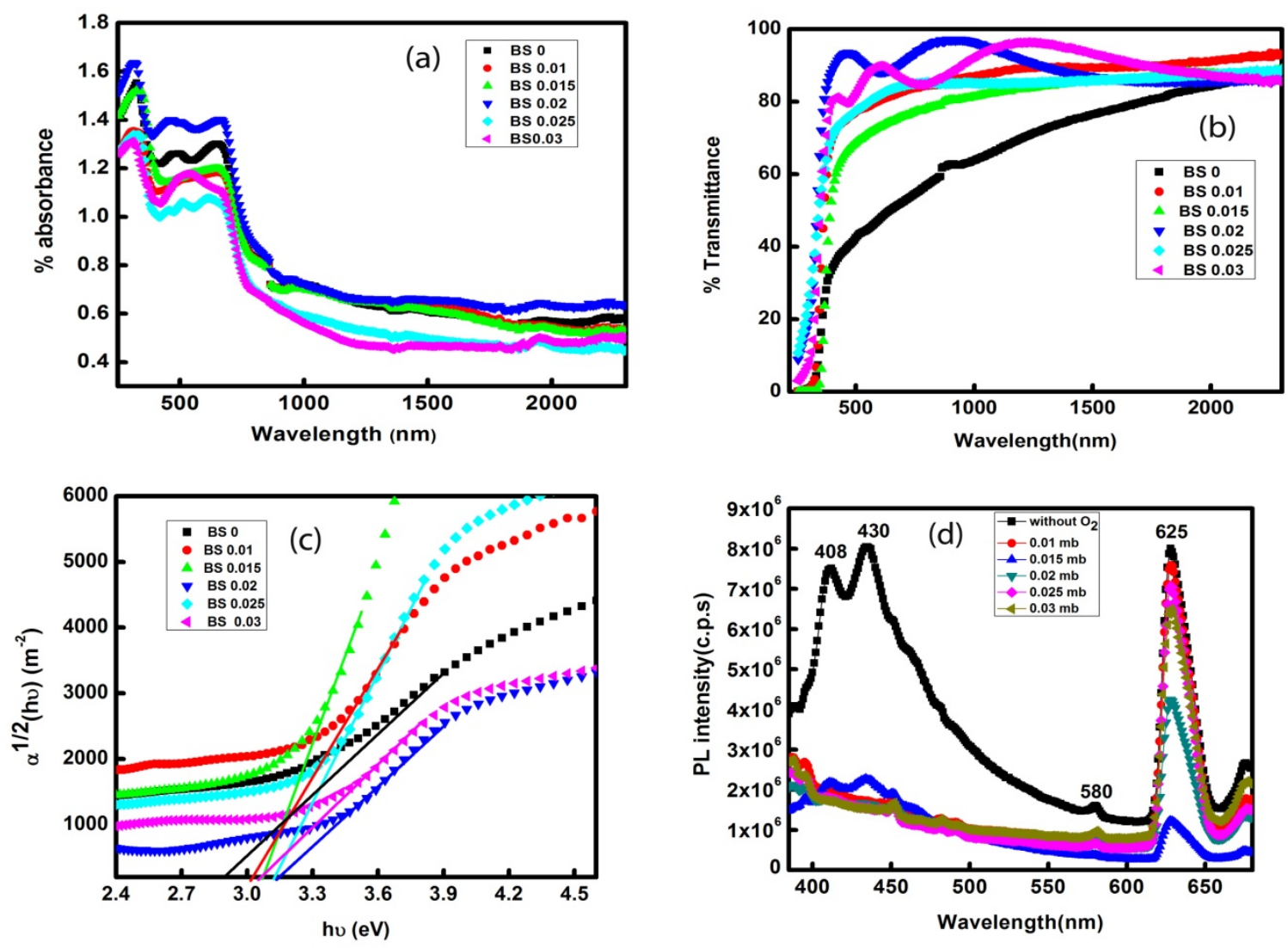

Fig.6. UV-Photoluminescence spectra of laser ablated $\mathrm{BaSnO}_{3}$ films for different oxygen pressures (a) absorbance spectra,(b) transmittance spectra, (c). Tauc plot and (d). Photoluminescence spectra.

The photoluminescence spectra of the $\mathrm{BaSnO}_{3}$ films recorded for an excitation wavelength $350 \mathrm{~nm}$ are given in Fig 6(d). In $\mathrm{BaSnO}_{3}$ films the emissions are caused by the transitions between energy levels of constituent elements barium and tin of the compound [44]. A violet emission around $400 \mathrm{~nm}$ can be expected in this compound due to direct transition between the energy levels of O2p and Sn5s [45]. Sumithra et al [46] observed blue-green emission around $445 \mathrm{~nm}$ and they have attributed this to the charge transfer transitions of $\mathrm{SnO}_{6}$ octahedra. They also reported a green emission around $550 \mathrm{~nm}$ due to the defect centres, or can be due to inter site positions leading to the formation of oxygen vacancy [46]. In the PL spectrum of the BS 0 film presents three intense emissions at 408, 430, 580 and 625 $\mathrm{nm}$. The violet emission at $408 \mathrm{~nm}$ can be due to ${ }^{2} \mathrm{P}^{0}{ }_{1 / 2}-{ }^{2} \mathrm{D}_{3 / 2}$ transitions of barium [47] or due to the transition between O2p and Sn5s levels [45]. The emission at $430 \mathrm{~nm}$ can be due to the charge transfer transitions in $\mathrm{SnO}_{6}$ octahedron [ 46] or due to ${ }^{3} \mathrm{P}_{0}{ }^{0}{ }^{3} \mathrm{P}_{1}$ transition of barium [47]. Soumitra et al., reported assigned this red emission in the $625 \mathrm{~nm}$ is due to the transition related to the oxygen interstitials and also due to lattice distortion along the preferred axis. The 
weak yellow emission at $580 \mathrm{~nm}$ may be due to the transition of the defect centres. Various authors assigned different reasons for the origin of red emission around $625 \mathrm{~nm}$. Upendra kumar et al reported the intense red emission in 625 nm region is due to the ${ }^{3} \mathrm{D}_{3}-{ }^{3} \mathrm{D}^{0}{ }_{3}$ transition of barium [44]. Soumitra et al., reported the intense red emission in 625 nm region is due to the ${ }^{3} \mathrm{D}_{3}-{ }^{3} \mathrm{D}^{0}{ }_{3}$ transition of barium [46]. The luminescence intensity in the spectrum shows that the intensity of the films deposited without oxygen pressure has high intensity in the blue emission region and the intensity decreases with increasing background pressure. The reduction in intensity of the films with oxygen ambience may be due to the reduction of defects or oxygen vacancies present in the sample.

\section{Conclusion}

The $\mathrm{BaSnO}_{3}$ films at different background oxygen pressures were prepared by pulsed laser deposition method. The XRD patterns show polycrystalline nature with cubic phase for all films. The film deposited at the oxygen pressure 0.02 mbar has the highest intensity peak with least FWHM, indicating the highest crystallinity for the films deposited at 0.02 mbar. The particle size also increases with increase with increase in oxygen pressure and lattice constant are calculated. The deterioration of intensity at higher oxygen pressure is due to enhanced scattering of the ejected particles in the laser produced plasma by the oxygen gas molecules. The band gap energy of the films are calculated. Morphological analysis shows that the films deposited at 0.02 mbar oxygen pressure has a uniform distribution of grains with defined grain boundary. All films show PL emission in the visible region. XPS analysis reveals that the oxidation state of tin as $\mathrm{Sn}^{4+}$ and the existence of oxygen vacancy in the prepared $\mathrm{BaSnO}_{3}$ films.

\section{Conflict of interest}

There is no conflict of interest in the study for any of the authors.

\section{References}

1] E. Moreira, J. M. Henriques, D. L. Azevedo, E. W. S. Caetano, V. N. Freire, U. L. Fulco, and E. L. Albuquerque JJ. Appl. Phys,112, 043703 (2012).

2] S Upadhyay, O. Parkash1 and D. Kumar,J. Phys. D: Appl. Phys. 37 1483, (2004).

3] J. Cerda, J. Arbiol, R. Diaz, G. Dezanneau, J.R. Morante, Mat. Lett .56 131- 136 (2002).

4] B. Ostrick, M. Fleischer, U. Lampe, H. Meixner, Sens. Actuators, B 44 601- 606 (1997).

5] C. Doroftei, P. D. Popa, F. Iacomi, J. Of OptoAndAdv Mater 14, No. 3-4, 413 - 417(2012).

6] Q. Liu, J. Dai, Z.Liu, X. Zhang, G. Zhu and G. Ding,J. Phys. D: Appl. Phys. 43455401 (2010).

7] R. M. Katiliute, P. Seibutas, M. Ivanov,R. Grigalaitis, A. Stanulis, J. Banys,And A. Kareiva,Ferroelectrics, 464:4958, (2014).

8] W.J Lee, H. J. Kim, J. Kang, D. H Jang, T.H Kim, J. H Lee, and K. H Kim, Annu. Rev. Mater. Res. 47:391-423 (2017).

9] B. Liu, Q Liu, Y. Zhang, Z. Liu, Lei Geng, J. of Alloys and Compounds 680 343-349(2016). 
10]S. A. Salehizadeh, H. M. Chenari, M. Shabani, H. A. Ahangar, R. Zamiri, A. Rebelo, J. Suresh Kumar, M. P. F. Graca and J. M. F. Ferreira, RSC Adv. 8, 2100(2018).

$11]$ M. Jawed Ansaree\& S Upadhyay, Ionics 21:2825-2838(2015).

12] Lampe U, Gerblinger J, Meixner H B 24-25: 657-660(1995).

13] Wensheng Lu Æ Helmut Schmidt, J Mater Sci 42:10007-10013, (2007) .

14] S. Upadhyay, Om Parkash, J. of Mater. Science Letters 16,1330-1332(1997).

15] Kutty TRN, Vivekanadan, R Mat Res Bull 22:1457 (1987).

16] H. J. Kim et al, Appl. Phys. Express. 5, 061102 (2012).

17] M. A. Pen , J. L. G. Fierro, Chem. Rev. 2001, 101, 1981 (2017).

18] S. Sallis, D. O. Scanlon, S. C. Chae, N. F. Quackenbush, D. A. Fischer et al, Appl. Phys. Lett. 103, 042105 (2013).

19] W.Lee, HyungJoon Kim, J Kang, D Hyun Jang, Tai Hoon Kim, J Hyuk Lee, and K H Kim ,Ann. Rev. of Mat Research 47, 391 (2017).

20] H.Kim, J.S. Horwitz, G.P.Kushto, Z. H.Kafafi, D.B.Chrisey, Appl.Phys.Lett.79 284(2001).

21] K.K. James, ArunAravind, M.K.Jayaraj, App Surf. Science 282,121- 125 (2013).

22] K.K. James, P.S. Krishnaprasad, K. Hasna, M.K. Jayaraj, J.ofPhy and Chem. of Solids 7664-69(2015).

23] B.C. Luon, J.Zhang,J.Wang,P.X.Ran, Ceramics International 412668-2672(2015).

24] V. Jayasree , R. Ratheesh, V. Ganesan , V.R. Reddy, C. Sudarsanakumar , V.P. MahadevanPillai , V.U. Nayar Thin Solid Films 517 603-608 (2008).

25]BassamAbdallaha, Abdul Kader Jazmatia, RaedaRefaaia, Materials Research. (2016).

26] Wang Zhaoyang, Hu Lizhong, Vacuum 83 906-909(2009).

27] D.B Cullity, Elements Of X-Ray Diffraction, (Addison-Wesley Inc., Massachusetts) (1956).

28].ShailUpadhyayBull. Mater.Sci., Vol. 36, No. 6, 1019-1036 (2013).

29] J. Cerda, J. Arbiol, R. Diaz, G. Dezanneau, J.R. Morante, Sens. Actuators, B 84 21-25 (2002).

30] R.S. Roth, J. Res. Natl. Bur. Stand. 58 (2) 75 (1957).

31] G.K. Williamson, W.H. Hall, Acta Metall. 22-31(1953).

32] S.R. Chalana, R. Jolly Bose, R. Reshmi Krishnan, V.S. Kavitha, R. SreejaSreedharan, V.P. MahadevanPillai J. of Phy and Chem. of Solids 95 24-36(2016).

33] A.K. Tripathi, M.C. Mathpal, M.K. Singh, P. Kumar, M.A.G. Soler, A. Agarwal, J. Alloy. Compd. 622 37-47 (2015).

34]M.R. Manju, K.S. Ajay, Noel M. D'Souza, S Hunagund, R.L.Hadimani, V.Dayal,J. Magn. Magn. Mater. (2017).

35] P. Singh, B.J. Brandenburg, Peter S., Prakash Singh, S Singh,D Kumar and Om Prakash; Jpn. J. Appl. Phys., 47 3540-3545(2008).

36] M. Kwoka, L. Ottaviano, M. Passacantando, S. Santucci, G. Czempik and J. Szuber, Thin Solid Films 490, 3642(2005).

37]J. Huang, Y Liu, Y. Wu, X. Li,Am J AnalytChem, 8, 60-71 (2017).

38]FulanZhong, H.Zhuang, QuanGu and J.Long ,RSCAdv, pp. 42474- 42481,6 (2016).

39] P Nithyadharseni, M.V Reddy, K.I Ozoemena, F.I Ezema, R.G Balakrishna, B.V. R

Chowdaria, J. Electrochem. Soc, 163, A540-A545 (2016).

40]Chuen-Lin Tien, Hong-Yi Lin, Chih-Kai Chang, and Chien-Jen Tang,Adv Cond Matter Phys, 26 47282, (2018).

41] S. katlakunta, K. praveena , R. singh, Materials Science-Poland, 31(4), , 581-586 (2013).

42] J.I. Pankove, Optical Processes in Semiconductors, Dover Publications, New York, (1971).

43] S.P. Patel, J.C. Pivin, V.V. Siva Kumar, A. Tripathi, D. Kanjilal, L. Kumar, Vacuum85 307-311(2010).

44] U. Kumar, Md. J. Ansaree, S. Upadhyay, Processing and Application of Ceramics 11 [3] 177-184,(2017).

45]S. Sumithra, N. Victor Jaya, J Supercond Nov Magn https://doi.org/10.1007/s10948-017-4504-8, (2017).

46] M. Ashokkumar, S. Muthukumaran Powder Technology 258 157-164,(2014).

47] A.S. Deepa, S. Vidya, P.C. Manu, Sam Solomon, Annamma John, J.K. Thomas, J. Alloys Compd. 509 18301835(2011).

48]E. Sansonetti, W.C. Martin, J. Phys. Chem. Ref. Data, 34 (4) 1559-2259 (2005). 